\title{
Electromagnetic freezing: Effects of weak oscillating magnetic fields on crab sticks
}

\author{
Laura Otero ${ }^{1}$, Miriam Pérez-Mateos, Antonio C. Rodríguez, and Pedro D. Sanz \\ Institute of Food Science, Technology and Nutrition (ICTAN-CSIC). c/ José Antonio Novais, 10, \\ 28040 Madrid, Spain
}

\section{ABSTRACT}

Since the earlier 2000s, electromagnetic freezers have been sold all over the world. According to the manufacturers, the oscillating magnetic fields (OMFs) applied by these devices are capable of avoiding ice damage in frozen foods. To assess the effectiveness of OMFs in preserving food quality, we froze crab sticks in a commercial electromagnetic freezer, both with ( $<2 \mathrm{mT}, 6-59 \mathrm{~Hz}$ ) and without OMF application. Crab sticks were also frozen in a conventional freezer, both with static- and forced-air conditions, to compare electromagnetic freezing with conventional methods. After $24 \mathrm{~h}$ and $1,3,6,9$, and 12 months of storage, we did not find any effect of the OMFs on the drip loss, water-holding capacity, toughness, and whiteness of the crab sticks frozen in the electromagnetic device. Moreover, no advantage of electromagnetic freezing over air-blast freezing was detected at the conditions tested. More experiments at larger magnetic field strength and wider frequency ranges are needed to have a complete view of the potential effects of OMFs on food freezing.

Keywords: electromagnetic freezing; oscillating magnetic fields; frozen storage; food preservation; crab sticks; food quality

\section{INTRODUCTION}

In the last few years, electromagnetic freezing has received much attention both from the food industry and from scientific circles (James, Purnell, \& James, 2015a; James, Purnell, \& James, 2015b; Kobayashi \& Kirschvink, 2014; Otero, Rodríguez, Pérez-Mateos, \& Sanz, 2016). Electromagnetic freezing basically involves applying a magnetic field during freezing and, thus, electromagnetic freezers simply consist of a magnetic field generator attached to a conventional quick-freezing unit. Since the earlier 2000s, some companies have patented and marketed

\footnotetext{
${ }^{1}$ Corresponding author: Tel.: +34 9154923 00; fax: +34 915493627.

E-mail address: I.otero@ictan.csic.es (L. Otero).
} 
electromagnetic freezers that apply different types of magnetic fields to theoretically improve the quality of frozen food. Thus, ABI Co., Ltd. (Chiba, Japan) sells 'CAS (Cells Alive System) freezers' that combine static and oscillating magnetic fields, while Ryoho Freeze Systems Co., Ltd. (Nara, Japan) commercializes 'Proton freezers' that use static magnetic fields and electromagnetic waves (ABI Co., 2007; Ryoho Freeze Systems Co., 2011).

According to the patents, oscillating magnetic fields (OMFs) applied during freezing enhance water supercooling, inhibit ice crystallization, and accelerate heat transfer (Owada, 2007; Owada \& Kurita, 2001; Owada \& Saito, 2010). When freezing occurs, either by lowering the temperature well below the freezing point or by ceasing the OMFs, small ice crystals are supposed to be formed throughout the whole volume of the product. In this way, damage produced in frozen foods is hypothetically reduced and, therefore, manufacturers claim that foods frozen in electromagnetic freezers maintain the quality of the fresh product unaltered. However, the extremely low strength of the OMFs commonly applied in commercial freezers (< $2 \mathrm{mT}$ ) casts doubt on the effects that these weak OMFs can have on a substance with a low magnetic susceptibility such as water. Moreover, the mechanisms adduced in the patents to explain the effects of OMFs on water molecules are vague and they have not been scientifically proved (Otero et al., 2016).

In a attempt to make the effects of weak OMFs on food freezing clear, Suzuki et al. (2009) and Watanabe, Kanesaka, Masuda, and Suzuki (2011) froze several food products, both with and without OMF application $(0.5 \mathrm{mT} / 50 \mathrm{~Hz})$. They did not find any effect of the oscillating magnetic field on the degree of supercooling and the freezing times recorded. Moreover, the ice crystals (size and shape), microstructure, drip losses, color, texture, and sensory evaluation were similar in all the frozen products. It is important to note that Suzuki et al. (2009) and Watanabe et al. (2011) performed all their experiments in a lab prototype and, therefore, this brings up the question of whether the characteristics of the OMF applied were exactly the same as those employed in commercial freezers. This is not easy to know because manufacturers usually do not provide these technical data (presence of static, oscillating, or both magnetic fields; strength and frequency values; combination with electric fields, electromagnetic waves, and so on).

To avoid this inconvenience, some authors have compared the quality of several foods frozen in both commercial electromagnetic freezers and conventional devices (Choi, Ku, Jeong, Jeon, \& Kim, 2015; Erikson et al., 2016; Kim et al., 2013b; Yamamoto, Tamura, Matsushita, \& Ishimura, 2005). Unfortunately, the few existing studies provide little or no information about the characteristics of the magnetic fields applied. Moreover, most of the experiments have been performed at very low temperatures, that is, at $-45^{\circ} \mathrm{C}$ and lower. At these conditions, the quality of frozen foods is usually well preserved with conventional methods and, therefore, observing improvements due to the OMF application could be difficult. Furthermore, an added obstacle is the inherent variability of food products (size, shape, structure, and composition) that can also contribute to diffuse the OMF effects. Thus, Yamamoto et al. (2005) compared the quality of 
chicken breasts frozen, at $-45^{\circ} \mathrm{C}$, in both a conventional rapid freezer and a CAS freezer. After one week of storage at $-30^{\circ} \mathrm{C}$, no differences were detected between the samples. Likewise, Erikson et al. (2016) compared the quality of gutted Atlantic cod frozen either in a CAS freezer at $-45^{\circ} \mathrm{C}$, in an air-blast freezer at $-35^{\circ} \mathrm{C}$, or in a cold storage room at $-30^{\circ} \mathrm{C}$. Even though the freezing rates achieved in each device were very different, the authors only found minor differences among the samples. By contrast, Kim et al. (2013a), Kim et al. (2013b), Ku et al. (2014), and Choi et al. (2015) froze beef, pork, and chicken samples in both a CAS freezer at $-55^{\circ} \mathrm{C}$ and an air-blast freezer at $-45{ }^{\circ} \mathrm{C}$. They concluded that electromagnetic freezing reduced the total freezing times and preserved the quality attributes of the samples better than air-blast freezing. However, the temperatures of the electromagnetic and the conventional freezer were too different to attribute these improvements exclusively to the OMF application.

To correctly discern the effect of OMFs, freezing experiments, with and without OMF application, should be performed in the same device. This is the only way to exclude the effect of other variables than the OMF application (freezing temperature, air convection, sample location in the freezer, and so on) on the results. In this sense, James, Reitz, and James (2015c) compared the freezing curves of garlic bulbs frozen with (0.1-0.4 mT) and without OMF application in a CAS freezer. They did not find any effect of the OMFs on the supercooling reached in the samples or on the freezing kinetics. Lamentably, to the best of our knowledge, no studies exist in the literature that analyze the effect of OMFs on food quality in such a way in commercial electromagnetic freezers.

To tackle all the problems described above, we characterized the magnetic fields produced in a commercial CAS freezer when programming different working conditions. To do so, we measured the magnetic field frequency and/or strength values at different locations in the freezing cabinet. Once magnetic fields were characterized, we performed freezing experiments, at $-25^{\circ} \mathrm{C}$, in this CAS freezer at three relevant OMF conditions and also without OMF application. To minimize the blurring effect of the inherent variability of food products on the results, we froze crab sticks of fixed size, shape, and composition. Crab sticks were also frozen in a conventional freezer at $-25^{\circ} \mathrm{C}$, both with static- and forced-air conditions, to compare electromagnetic freezing with conventional methods. After $24 \mathrm{~h}$ and 1, 3, 6, 9, and 12 months of storage, drip loss, water-holding capacity, toughness, and whiteness were compared in fresh and frozen crab sticks to assess the efficacy of OMFs in preserving food quality.

This paper provides valuable information for evaluating the effectivity of OMFs in improving the quality of frozen foods just after freezing and also after long-term frozen storage. In this way, it increases the knowledge on electromagnetic freezing, an innovative technology already implemented for industrial food freezing, but scientifically unexplored. 


\section{MATERIAL AND METHODS}

\subsection{Sample}

111 Fresh crab sticks, all produced in the same batch, were acquired to a Spanish manufacturer.

112 According to the product label, the main ingredients of the crab sticks were surimi (44\%), water, 113 starch, modified starch, sunflower oil, salt, and egg albumen.

114 After reception, all the sticks were unpacked and cut in half. The portions obtained (about 38 $115 \mathrm{~mm}$ length, $15 \mathrm{~mm}$ width, and $15 \mathrm{~mm}$ height) were packed in plastic bags and stored at $4{ }^{\circ} \mathrm{C}$ 116 before freezing.

\subsection{Magnetic freezer and its characterization}

Magnetic freezing experiments were carried out in a commercial CAS freezer (ABI Co., Ltd., Chiba, Japan). The freezer consisted of a cooling unit, 2 fans, a control panel, and a freezing cabinet (Figure 1a). This cabinet contained a rack with 10 equidistant rails to place up to 10 trays for food freezing and the magnetic field generators.

The CAS freezer was equipped with both static and oscillating magnetic field generators to assist the freezing process. The static magnetic field was produced by a number of permanent magnets embedded in the front door and in the ceiling, the floor, and the left and rear walls of the freezing cabinet, while the OMF was generated by 4 rectangular magnetic coils located inside the cabinet. These coils (160 cm height, $70 \mathrm{~cm}$ length) were arranged around the food trays and they were separated from each other by a distance of $18 \mathrm{~cm}$.

Different freezing conditions, namely, air temperature (down to $-50{ }^{\circ} \mathrm{C}$ ), airflow $(0-100 \%)$, and 'CAS energy' (0-100\% CAS), could be set at the control panel. Unfortunately, the precise values associated to these airflow and CAS conditions were not provided by the freezer manufacturer. To know these values, measurements of air velocity, magnetic field strength, and frequency were performed for $100 \%$ air flow and different CAS conditions at several locations in the freezing cabinet.

Air velocity measurements were carried out by using an anemometer (VT100, Kimo S.A., Montpon, France). These measurements were performed at the center of trays 1, 5, and 10 of the freezing cabinet (Figure 1a). At these same locations, and also, at the middle of the four edges of each tray (points b, c, d, and e in Figure 1b), magnetic field strength was evaluated for both static and oscillating magnetic fields by using a teslameter (GM07, Hirst Magnetic Instruments LTD, Falmouth, UK), while a current probe (TCP202A, Tektronix Inc., Beaverton, OR, USA), an oscilloscope (TDS3012B, Tektronix Inc., Beaverton, OR, USA), and a circular antenna (frequency probe) were used to determine the OMF frequency. The $\mathrm{X}, \mathrm{Y}$, and $\mathrm{Z}$ 
rectangular components of the magnetic field strength were measured separately and, then, summed vectorially to obtain the total magnetic field strength.

\subsection{Freezing experiments and storage}

147 Magnetic freezing experiments were performed in the CAS freezer described above. Crab sticks 148 were frozen at different conditions, both with $(10 \%, 50 \%$, and $100 \%$ CAS) and without (0\% 149 CAS) OMF application. In all these experiments, the crab sticks were located on tray 5 in the 150 freezing cabinet, that is, the tray situated approximately at the center of the magnetic coils. The 151 air temperature and air flow were fixed at $-25^{\circ} \mathrm{C}$ and $100 \%$, respectively.

152 Air-blast and static-air freezing experiments were carried out at $-25^{\circ} \mathrm{C}$ in a conventional freezer 153 (model 0-6373, AGA-Frigoscandia, Helsingborg, Sweden) by setting the air speed to the 154 maximum value $(4.8 \mathrm{~m} / \mathrm{s})$ and $0 \mathrm{~m} / \mathrm{s}$, respectively. In all these experiments, the crab sticks were 155 located approximately at the center of the freezing cabinet.

156 During the freezing process, the temperature evolution in the samples was measured by 1572 T-type thermocouples located at the thermal center of 2 crab sticks. Moreover, air temperature 158 was also monitored at 2 different locations in the freezer. Thermocouple measurements were 159 recorded every second by a data acquisition system (MW100, Yokogawa Electric Corp., Tokyo, 160 Japan).

161 The freezing process was considered completed when the thermal center of the samples 162 reached $-20^{\circ} \mathrm{C}$. Then, the crab sticks were taken out of the freezer and transferred to a cold storage warehouse at $-20^{\circ} \mathrm{C}$.

All the freezing experiments were performed in triplicate.

\subsection{Quality attributes}

167 Quality attributes were evaluated in both fresh and frozen crab sticks after $24 \mathrm{~h}$ (month 0), 1, 3, 1686,9 , and 12 months of frozen storage. Except for the drip loss measurements, the frozen crab 169 sticks were thawed for $24 \mathrm{~h}$ at $4{ }^{\circ} \mathrm{C}$ before the determinations.

\subsubsection{Drip loss}

171 In this study, the term 'drip' was used to describe the exudates from both fresh and frozen 172 samples after $24 \mathrm{~h}$ of storage at $4{ }^{\circ} \mathrm{C}$. Obviously, the frozen samples were thawed during this 173 storage period. 
For each determination, 6 crab sticks were weighed, packed in a plastic bag, and stored at $4{ }^{\circ} \mathrm{C}$. After $24 \mathrm{~h}$ of storage, the surface of the crab sticks was dried with soft paper and, then, the samples were weighed again.

Drip loss (DL) was expressed as the percent of mass loss according to Eq. (1):

$$
D L(\%)=\frac{\left(M_{b s}-M_{a s}\right)}{M_{b s}} \times 100
$$

where $M_{b s}$ and $M_{a s}$ are the masses $(g)$ of the sticks before and after the storage period, respectively.

For each experiment, the drip loss determinations were performed in duplicate.

\subsubsection{Water-holding capacity}

The water-holding capacity (WHC) of the crab sticks was measured by using centrifugal force to remove the free and loosely bound water from the samples. For each determination, $3 \mathrm{crab}$ sticks were coarsely chopped. Then, about $8 \mathrm{~g}$ of the chopped sticks was weighed and put into a centrifuge tube. The tube had a perforated disc, covered with 2 filter papers, and located approximately half way down the tube. The sample was placed on this perforated disc and centrifuged at $2200 \mathrm{xg}$ and $4{ }^{\circ} \mathrm{C}$ for $10 \mathrm{~min}$ (Sorvall Evolution RC centrifuge, model 728311 , Thermo Electron Corporation, Asheville, NC, USA). After centrifugation, the chopped sticks were weighed again. WHC was expressed as the percent of water retained per $100 \mathrm{~g}$ of water present in the sample prior to centrifuging according to:

$$
W H C(\%)=\left(1-\frac{\left(M_{b c}-M_{a c}\right)}{M_{b c} \times m_{t w}}\right) \times 100
$$

where $M_{b c}$ and $M_{a c}$ are the masses ( $g$ ) of the chopped sticks before and after centrifugation, respectively, and $\mathrm{m}_{\mathrm{tw}}$ is the mass fraction of total water present in the sample prior to centrifuging. $m_{t w}$ was evaluated in the samples by determining the mass loss in chopped crab sticks after oven drying at $105^{\circ} \mathrm{C}$ until a constant weight was reached. All WHC and $\mathrm{m}_{\mathrm{tw}}$ measurements were performed in triplicate.

\subsubsection{Toughness}

201 The toughness of the crab sticks was evaluated by a Warner-Bratzler test to determine the force needed to shear the sample. A Texture Analyser (TA-XTPlus, Stable Micro System Ltd., Surrey, UK), equipped with a V-shaped Warner-Bratzler blade and controlled by the Texture Exponent 32 software (v. 6.1.5.0), was employed. For each experiment, 6 crab sticks were sheared $(2 \mathrm{~mm} / \mathrm{s}$ crosshead speed, $5 \mathrm{~kg}$ load cell) perpendicular to the fibers and the maximum force $(\mathrm{N})$ was recorded.

\subsubsection{Whiteness}


The whiteness of the crab sticks was characterized objectively according to the $L^{*}, a^{*}$, and $b^{*}$ color parameters in the CIELab uniform color space defined by the Commission Internationale de l'Éclairage. To do so, a CM-3500d spectrophotometer managed by the color data software CM-S100w SpectraMagic ${ }^{\mathrm{TM}}$ (Konica Minolta, Tokyo, Japan) was employed. The illuminating and viewing configurations of the instrument complied with the CIE diffuse $/ 8^{\circ}$ geometry. The spectrophotometer operated in the reflectance specular included mode and the measuring aperture was $8 \mathrm{~mm}$ in diameter. Measurements were made with the D65 standard illuminant and a ten-degree observer angle. The instrument was calibrated with black and white standards before each series of analysis.

For each experiment, whiteness was evaluated in 3 crab sticks. Before the measurements, the orange outer layer of the crab sticks was carefully removed. Two measurements were performed in each sample (one at the center of the upper side of the crab stick and the other at the center of its lower side) and the obtained $L^{*}, a^{*}$, and $b^{*}$ values were averaged. From these mean values, the whiteness index of each sample was calculated according to:

$$
\text { Whiteness }=100-\sqrt{\left(100-L^{*}\right)^{2}+a^{* 2}+b^{* 2}}
$$

\subsection{Statistical analysis}

The statistical analysis of the data was performed using IBM SPSS Statistics v. 22.0.0.1 for Windows (IBM Corp., Armonk, NY, USA).

At month 0 , a multivariate step-wise linear discriminant analysis was carried out to determine whether the fresh crab sticks and those frozen by different methods can be distinguished and, in this case, which quality attributes are the best to explain the differences among them.

To test the main effects of the freezing conditions and the storage time on the quality attributes of the thawed crab sticks, a two-way analysis of variance (ANOVA) was performed on the data by using the General Linear Model procedure of the statistical software. The significance level was set at $5 \%$. A Tukey-b test was applied for post-hoc comparisons.

\section{RESULTS AND DISCUSSION}

\subsection{Magnetic freezer characterization}

Air velocity $(\mathrm{m} / \mathrm{s})$ and magnetic field strength $(\mathrm{mT})$ and frequency $(\mathrm{Hz})$ values were measured at several locations in trays 1,5 , and 10 of the freezing cabinet after programming $100 \%$ air flow and different 'CAS energy' conditions (0-100\%). 
Air velocity at different locations strongly depended on the relative position from the fans of the freezer. Thus, the maximum air velocity was measured at the center of tray 5 , while the minimum value was registered for tray 10 . At the center of tray 5 , air velocity increased from 0 $\mathrm{m} / \mathrm{s}$ for $0 \%$ air flow up to $3.8 \mathrm{~m} / \mathrm{s}$ for $100 \%$ air flow.

Magnetic field strength values at different locations depended on the distance to the permanent magnets and to the magnetic coils as expected. Thus, for a given tray, the X-component of the static magnetic field was larger at the front and back edges of the tray (positions $d$ and $e$ in Figure 1b) because of the front-door and rear-wall magnets, while the Y-component was larger at the left edge because no permanent magnets exist on the right side of the freezing cabinet (Figure 1a). Moreover, the Z-component of the static field was larger on trays 1 and 10 than on tray 5 due to the ceiling and floor magnets. In a similar way, the oscillating magnetic field was not uniform throughout the freezing cabinet, but it depended on the relative location from the magnetic coils. In general, the $\mathrm{X}$ - and $\mathrm{Z}$ - components of the OMF were larger than the $\mathrm{Y}$ component at the positions measured in each tray. For a given tray, the $\mathrm{X}$-component was maximum at the front edge (position $d$ in Figure $1 b$ ), while both the $Y$ - and Z-components presented a minimum at this location. The lowest $X$-values were measured at the middle of the tray (positions b, a, and c in Figure 1b). At the center (position a in Figure 1b), the Z-component of the oscillating magnetic field was maximum, whereas the maximum $Y$-values were found at the left and right edges of each tray (positions $b$ and $c$ in Figure 1b).

Furthermore, it is important to note that the OMF also depended on the 'CAS energy' conditions programmed at the control panel. Thus, at 0\% CAS, no OMF was applied in the freezer and only the static magnetic field acted. For other CAS conditions, different OMFs were produced. Figure 2 shows the X-component of the OMF measured at the center of tray 5 for different 'CAS energy' conditions. It clearly shows that the magnetic field strength increased from $0 \%$ to $10 \%$ CAS and, then, slightly decreased for growing 'CAS energy' values, while frequency increased linearly from $0 \%$ to $100 \%$ CAS.

The freezing experiments described in this paper were performed at different conditions, both with (10\%, 50\%, and $100 \%$ CAS) and without (0\% CAS) OMF application, to evaluate the effect of OMFs on the quality of the frozen crab sticks. Table 1 shows the corresponding OMF strength and frequency values measured at the center of tray 5 and the maximum and minimum values registered all over this tray. Table 1 also includes the strength of the static magnetic field, induced by the permanent magnets, at this same tray. Unfortunately, the effects of the static magnetic field alone on the quality of crab sticks could not be assessed in this paper because the permanent magnets were embedded in the freezer and, therefore, they could not be removed to make comparisons. product 
Representative freezing curves for conventionally (static air and air blast) and CAS ( $0 \%$ and $100 \%$ ) frozen crab sticks are depicted in Figure 3 . The curves clearly show the 3 key steps of the freezing process: precooling, phase transition, and tempering. During the phase transition step, the temperature remained constant at about $-3^{\circ} \mathrm{C}$, that is, at the initial freezing point of the crab sticks while the latent heat of crystallization was removed. In this phase, most of the ice crystals are formed in the product and, therefore, the rate of heat removal is crucial for the quality of the frozen food. The slower the heat removal, the larger the ice crystals formed and, therefore, the poorer the quality of the product (Kiani \& Sun, 2011). Figure 3 reveals that the rate of heat removal was significantly slower in the static-air freezing experiments as expected. Thus, the characteristic freezing time (time needed to change the temperature at the center of the sample from the initial freezing point to a temperature $10^{\circ} \mathrm{C}$ lower) was $74.9 \pm 4.5 \mathrm{~min}$; that is, about 5 times longer than that corresponding to the rest of methods. By contrast, no significant differences were found among CAS and air-blast experiments $(14.4 \pm 0.7,13.0 \pm 0.5$, and $16.0 \pm 0.8 \mathrm{~min}$ in $0 \% \mathrm{CAS}, 100 \% \mathrm{CAS}$, and air-blast freezing experiments, respectively).

To evaluate the effectiveness of OMFs in retaining the quality of the fresh product, drip loss, water-holding capacity, toughness, and whiteness were measured in fresh and frozen-thawed samples after $24 \mathrm{~h}$ of storage (month 0 in Figure 4). The data clearly proved that all the frozen samples, whichever the freezing method employed, significantly differed $(p<0.05)$ from the fresh crab sticks. The multivariate stepwise linear discriminant analysis of the data revealed that the water-holding capacity $(F$ value $=76.13)$ and drip loss $(F$ value $=24.67)$ were the properties that best discriminated among the different samples (fresh, CAS, air-blast, and static-air frozen) at month 0 . Figure 5 illustrates how the water-holding capacity allowed a perfect discrimination between fresh and frozen samples, while the drip loss discriminated among samples frozen at different conditions to a lesser extent. In general, samples frozen in static air showed the highest drip loss, while air-blast frozen sticks produced the lowest exudates after thawing. Drip losses in CAS frozen samples presented intermediate values and no effect of the OMF application was detected.

Thus, in contrast to the claims stated in patents and commercial advertisements (Owada, 2011), our results revealed that OMFs failed to avoid damage caused by ice crystals and, therefore, they were not able to maintain the quality attributes of the fresh crab sticks intact after thawing. Thus, the drip loss and toughness of electromagnetically frozen samples were significantly larger than those of the fresh crab sticks, while the WHC was significantly lower. Whiteness was the only quality attribute that remained unaltered after thawing, but it is interesting to note that the same occurred for all the freezing methods tested.

\subsection{Effect of freezing conditions on quality attributes during frozen storage} samples after $24 \mathrm{~h}$ and $1,3,6,9$, and 12 moths of storage to evaluate the effect of the freezing 
conditions (0\% CAS, 10\% CAS, 50\% CAS, 100\% CAS, air-blast, and static-air freezing) on the quality of the crab sticks (Figure 4).

The statistical analysis of the data showed that both the freezing conditions (FC) and the storage time $(t)$ significantly affected $(p<0.05)$ all the quality attributes (Table 2$)$. The effect of the freezing conditions was especially important for the drip loss ( $F$ value $=399.93$ ), while the effect of the storage time was more relevant for all the other quality attributes. Moreover, a significant interaction between the freezing conditions and the storage time $(F C \times t)$ was found for drip loss, WHC, and toughness. Therefore, the evolution of these quality attributes during storage was different in samples frozen by different methods.

No effect of the OMFs applied, whichever their strength or frequency, was found in any of the quality attributes of the crab sticks. Thus, post-hoc comparisons after the two-way ANOVA did not detect significant differences between the crab sticks frozen with or without OMFs in the CAS freezer. Similar results were reported by Suzuki et al. (2009) and Watanabe et al. (2011) who did not find any effect of OMFs on the microstructure, drip losses, color, texture, and sensory evaluation of frozen radish, sweet potato, spinach, yellow tail fish and tuna. Likewise, Yamamoto et al. (2005) did not detect apparent effects of the OMF conditions (1.5-2 mT at 20, 30 , and $40 \mathrm{~Hz}$ ) on the drip and cooking losses and the rupture stress and strain of chicken breasts frozen in a CAS freezer, after one week or six months of frozen storage. In this sense, James et al. (2015a) declared they had not found clear and repeatable effects of the CAS conditions on the quality (dimensions, weights, drip loss, color, moisture content, sugar content, and texture) of magnetically frozen fruit, vegetables, meat, and fish products.

The statistical analysis of the results revealed significant differences between the crab sticks frozen in the CAS freezer and those frozen by conventional methods. Thus, the samples frozen in static air presented the largest drip loss and toughness and the lowest water-holding capacity and whiteness. By contrast, air-blast frozen samples released the lowest drip after thawing and exhibited the largest WHC. Toughness and whiteness in these samples were similar to those observed in CAS frozen crab sticks. Therefore, we did not find any advantageous effect of CAS freezing over conventional air-blast freezing at the conditions tested.

The results described above are consistent with the thermal kinetics observed in the freezing curves. Thus, the slow freezing rates achieved in the static-air freezing experiments allowed water molecules to migrate and agglomerate, forming large ice crystals. During frozen storage, recrystallization phenomena occurred that increased ice damage. It is well-known that, in fish gels, large ice crystals produce the dehydratation of the gel network, affect protein interactions, and induce starch retrogradation. All these phenomena greatly affect the physical attributes of crab sticks and produce quality losses (Kato, Lee, Fukuda, \& Arai, 1993; Kolbe, 2000; Park \& Beliveau, 2014). By contrast, the much quicker freezing rates in all the other experiments reduced water migration and, thus, smaller ice crystals were formed that produce significantly lower quality losses on thawing. 


\section{CONCLUSIONS}

The oscillating magnetic fields $(<2 \mathrm{mT}, 6-59 \mathrm{~Hz}$ ) applied during freezing did not avoid ice

364 damage in crab sticks. Thus, $10 \%, 50 \%$, and $100 \%$ CAS frozen samples significantly differed

365 from the fresh crab sticks. These OMFs not only were incapable of avoiding ice damage but

366 also had no effect, whichever their strength or frequency, on the quality attributes of the

367 samples after thawing. Thus, no significant differences were detected between samples frozen

368 with (10\%, 50\%, and 100\% CAS) and without (0\% CAS) OMF application. In this sense, it is

369 important to note that the OMF strength tested in the experiments of this paper was lower than

$3702 \mathrm{mT}$, that is, only two orders of magnitude larger than the Earth's natural magnetic field (0.025-

$3710.06 \mathrm{mT})$. Moreover, the frequency range studied was also rather narrow $(6-59 \mathrm{~Hz})$. Even

372 though these are the OMF strength and frequency ranges usually employed in commercial CAS

373 freezers, it should be desirable to perform investigations at much more wide ranges to have a

374 complete view of the potential effects of OMFs on food freezing.

375

\section{Acknowledgments}

377 This work was supported by the Spanish Ministry of Economy and Competitiveness (MINECO)

378 through the project AGL2012-39756-C02-01. Antonio C. Rodríguez acknowledges a predoctoral

379 contract from MINECO, jointly financed by the European Social Fund, in the framework of the

380 National Program for the Promotion of Talent and its Employability (National Sub-Program for

381 Doctors Training). The authors thank Javier Sánchez-Benítez, researcher at Universidad

382 Complutense de Madrid (Facultad de Químicas), for his help in the electromagnetic freezer

383 characterization. They also thank Laura Barrios, head of the Statistical and Operational

384 Research Service of CSIC (Spain), for her advice in the statistical analysis of the data and

385 Ignacio Rodríguez, technician at ICTAN-CSIC, for his assistance in the lab work. 
ABI Co., L. (2007). CAS: Cells Alive System. The CAS energy function has an international patent. Accesed 2016 September http://www.rayswebstudio.co.uk/Lynton/download-pdf/English\%20Brochure_01.pdf.

Choi, Y. S., Ku, S. K., Jeong, J. Y., Jeon, K. H., \& Kim, Y. B. (2015). Changes in ultrastructure and sensory characteristics on electro-magnetic and air blast freezing of beef during frozen storage. Korean Journal for Food Science of Animal Resources, 35(1), 27-34.

Erikson, U., Kjørsvik, E., Bardal, T., Digre, H., Schei, M., Søreide, T. S., \& Aursand, I. G. (2016). Quality of Atlantic cod frozen in cell alive system, air-blast, and cold storage freezers. Journal of Aquatic Food Product Technology, 1-20.

James, C., Purnell, G., \& James, S. J. (2015a). Can magnetism improve the storage of foods? New Food, 18(2), 40-43.

James, C., Purnell, G., \& James, S. J. (2015b). A review of novel and innovative food freezing technologies. Food and Bioprocess Technology, 8(8), 1616-1634.

James, C., Reitz, B., \& James, S. J. (2015c). The freezing characteristics of garlic bulbs (Allium sativum L.) frozen conventionally or with the assistance of an oscillating weak magnetic field. Food and Bioprocess Technology, 8(3), 702-708.

Kato, N., Lee, N. H., Fukuda, K., \& Arai, K.I. (1993). Quality of frozen seafood analog such as crab leg type and scallop adductor type products. Nippon Suisan Gakkaishi, 59(5), 815820.

Kiani, H., \& Sun, D. W. (2011). Water crystallization and its importance to freezing of foods: A review. Trends in Food Science and Technology, 22(8), 407-426.

Kim, Y. B., Jeong, J. Y., Ku, S. K., Kim, E. M., Park, K. J., \& Jang, A. (2013a). Effects of various thawing methods on the quality characteristics of frozen beef. Korean Journal for Food Science of Animal Resources, 33(6), 723-729.

Kim, Y. B., Woo, S. M., Jeong, J. Y., Ku, S. K., Jeong, J. W., Kum, J. S., \& Kim, E. M. (2013b). Temperature changes during freezing and effect of physicochemical properties after thawing on meat by air blast and magnetic resonance quick freezing. Korean Journal for Food Science of Animal Resources, 33(6), 763-771.

Kobayashi, A., \& Kirschvink, J. L. (2014). A ferromagnetic model for the action of electric and magnetic fields in cryopreservation. Cryobiology, 68(2), 163-165.

Kolbe, F. (2000). Freezing technology. In J. W. Park (Ed.), Surimi and surimi seafood (pp. 167200). New York, Basel: Marcel Dekker.

Ku, S. K., Jeong, J. Y., Park, J. D., Jeon, K. H., Kim, E. M., \& Kim, Y. B. (2014). Quality evaluation of pork with various freezing and thawing methods. Korean Journal for Food Science of Animal Resources, 34(5), 597-603.

Otero, L., Rodríguez, A. C., Pérez-Mateos, M., \& Sanz, P. D. (2016). Effects of magnetic fields on freezing: Application to biological products. Comprehensive Reviews in Food Science and Food Safety, 15(3), 646-667.

Owada, N. (2007). Highly-efficient freezing apparatus and high-efficient freezing method. US Patent 7237400 B2. 03.07.2007. United States.

Owada, N. (2011). CAS technology: From storing fresh food to preserving medical resources. Organ Biology, 18(1), 71-78.

Owada, N., \& Kurita, S. (2001). Super-quick freezing method and apparatus therefor. US Patent 6250087 B1. 26.06.2001. United States.

Owada, N., \& Saito, S. (2010). Quick freezing apparatus and quick freezing method. US Patent 7810340B2. Oct. 12, 2010.

Park, J. W., \& Beliveau, J. L. (2014). Manufacture of crabsticks. In J. W. Park (Ed.), Surimi and surimi seafood (Third Edition ed., pp. 245-270). Boca Raton: CRC Press. Taylor \& Francis Group. 
Ryoho Freeze Systems Co., L. (2011). Accessed 2016 September 21. http://www.protongroup.net/en/case/index.html.

Suzuki, T., Takeuchi, Y., Masuda, K., Watanabe, M., Shirakashi, R., Fukuda, Y., Tsuruta, T., Yamamoto, K., Koga, N., Hiruma, N., Ichioka, J., \& Takai, K. (2009). Experimental investigation of effectiveness of magnetic field on food freezing process. Transactions of the Japan Society of Refrigerating and Air Conditioning Engineers, 26, 371-386. field on supercooling in food freezing. In International Institute of Refrigeration (Ed.), The 23rd IIR International Congress of Refrigeration: Refrigeration for sustanaible development. (Vol. 1, pp. 2892). Prague, Czech Republic.

Yamamoto, N., Tamura, S., Matsushita, J., \& Ishimura, K. (2005). Fracture properties and microstructure of chicken breasts frozen by electromagnetic freezing. Journal of Home Economics of Japan, 56(3), 141-151.

450 
TABLE 1 Magnetic field strength and frequency values measured at the center of tray 5 after programming different 'CAS energy' conditions in the CAS freezer. Values between parentheses represent minimum and maximum values measured all over the tray.

\begin{tabular}{|c|c|c|c|c|c|c|c|c|c|}
\hline & \multicolumn{8}{|c|}{$\begin{array}{l}\text { MAGNETIC FIELD STRENGTH } \\
\qquad(\mathrm{mT})\end{array}$} & \multirow{3}{*}{$\begin{array}{c}\text { MAGNETIC FIELD } \\
\text { FREQUENCY } \\
(\mathrm{Hz}) \\
\text { Oscillating } \\
\text { magnetic field }\end{array}$} \\
\hline & \multicolumn{4}{|c|}{ Static magnetic field } & \multicolumn{4}{|c|}{ Oscillating magnetic field } & \\
\hline & $x$ & Y & Z & Total & $x$ & $Y$ & Z & Total & \\
\hline $0 \%$ CAS & & & & & $\begin{array}{c}0 \\
(0-0)\end{array}$ & $\begin{array}{c}0 \\
(0-0)\end{array}$ & $\begin{array}{c}0 \\
(0-0)\end{array}$ & $\begin{array}{c}0 \\
(0-0)\end{array}$ & 0 \\
\hline $10 \%$ CAS & 0.07 & 0.12 & 0.04 & 0.14 & $\begin{array}{c}0.92 \\
(0.92-1.81)\end{array}$ & $\begin{array}{c}0.70 \\
(0.60-1.25)\end{array}$ & $\begin{array}{c}0.99 \\
(0.42-0.99)\end{array}$ & $\begin{array}{c}1.52 \\
(1.51-1.95)\end{array}$ & 6 \\
\hline $50 \%$ CAS & $(0.00-0.15)$ & $(0.02-0.22)$ & $(0.02-0.04)$ & $(0.12-0.22)$ & $\begin{array}{c}0.79 \\
(0.79-1.71)\end{array}$ & $\begin{array}{c}0.69 \\
(0.58-1.20)\end{array}$ & $\begin{array}{c}0.93 \\
(0.41-0.93)\end{array}$ & $\begin{array}{c}1.40 \\
(1.40-1.85)\end{array}$ & 30 \\
\hline $100 \%$ CAS & & & & & $\begin{array}{c}0.74 \\
(0.74-1.69)\end{array}$ & $\begin{array}{c}0.71 \\
(0.59-1.18)\end{array}$ & $\begin{array}{c}0.92 \\
(0.43-0.92)\end{array}$ & $\begin{array}{c}1.38 \\
(1.38-1.84)\end{array}$ & 59 \\
\hline
\end{tabular}


TABLE 2: Results of the two-way ANOVA for the effect of the freezing conditions $(0 \%$ CAS, $10 \%$ CAS, $50 \%$ CAS, $100 \%$ CAS, air-blast, and static-air freezing) and the storage time $(0-12$ months $)$ on the quality of crab sticks $(p<0.05)$.

\begin{tabular}{|c|c|c|c|c|}
\hline & Sources of variation & $\begin{array}{c}\text { Degrees } \\
\text { of } \\
\text { freedom }\end{array}$ & $\mathrm{F}$ & Sig \\
\hline \multirow{3}{*}{ Drip loss } & Freezing conditions (FC) & 5 & 399.93 & 0.00 \\
\hline & Storage time $(\mathrm{t})$ & 5 & 4.76 & 0.00 \\
\hline & $\mathrm{FC} \times \mathrm{t}$ & 25 & 13.13 & 0.00 \\
\hline \multirow{3}{*}{ Water-holding capacity } & Freezing conditions (FC) & 5 & 31.64 & 0.00 \\
\hline & Storage time $(\mathrm{t})$ & 5 & 747.54 & 0.00 \\
\hline & $\mathrm{FC} \times \mathrm{t}$ & 25 & 2.10 & 0.01 \\
\hline \multirow[t]{3}{*}{ Toughness } & Freezing conditions (FC) & 5 & 37.33 & 0.00 \\
\hline & Storage time $(\mathrm{t})$ & 5 & 1233.81 & 0.00 \\
\hline & $\mathrm{FC} \times \mathrm{t}$ & 25 & 3.47 & 0.00 \\
\hline \multirow[t]{3}{*}{ Whiteness } & Freezing conditions (FC) & 5 & 17.76 & 0.00 \\
\hline & Storage time $(\mathrm{t})$ & 5 & 93.73 & 0.00 \\
\hline & $\mathrm{FC} \times \mathrm{t}$ & 25 & 1.23 & 0.22 \\
\hline
\end{tabular}




\section{FIGURE CAPTIONS}

Figure 1 Schematic drawing of the CAS freezer: a) Main components, b) Points at which magnetic field measurements were performed in freezing trays 1, 5, and 10.

Figure 2 Characteristics of the oscillating magnetic field for different 'CAS conditions' in the magnetic freezer ( $\mathrm{BBI}$ Co., Ltd., Chiba, Japan): a) X-component of the magnetic field strength and b) frequency. Measurements were performed at the center of tray 5 in the freezing cabinet.

Figure 3 Representative freezing curves of crab sticks frozen at $-25^{\circ} \mathrm{C}$ and different conditions. (- -): 0\% CAS; (-): 100\% CAS; (-): Air blast, and ( $\cdots$ ): Static air.

Figure 4 (a) Drip loss, (b) water-holding capacity, (c) toughness, and (d) whiteness of fresh crab sticks $(\Delta)$ and of those frozen at different freezing conditions ( $\square: 0 \%$ CAS, $\square: 10 \%$ CAS, $\square: 50 \%$ CAS, $\mathbf{a}: 100 \%$ CAS, $\diamond:$ air blast, and - : static air) during storage at $-20^{\circ} \mathrm{C}$. Vertical bars represent standard error. For a given storage time, different letters indicate significant differences between means $(p<0.05)$ due to the freezing conditions. No letters indicate no significant differences between means.

Figure 5 Scatter plot of the crab sticks data at month 0 for the most discriminant quality attributes. $\triangle$ : Fresh, $\square: 0 \%$ CAS frozen, $\square: 10 \%$ CAS frozen, $\square: 50 \%$ CAS frozen, $\mathbf{\square}:$ 100\% CAS frozen, $\diamond$ : air-blast frozen, and $\bullet$ : static-air frozen crab sticks. 
FIGURE 1

a)

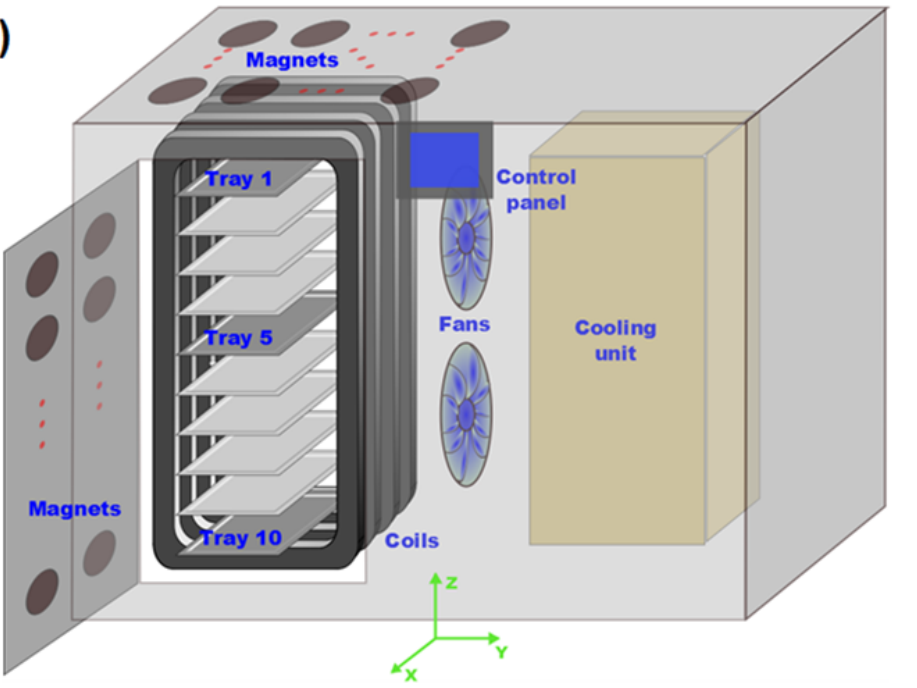

b)

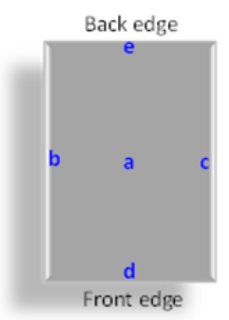


FIGURE 2

a)

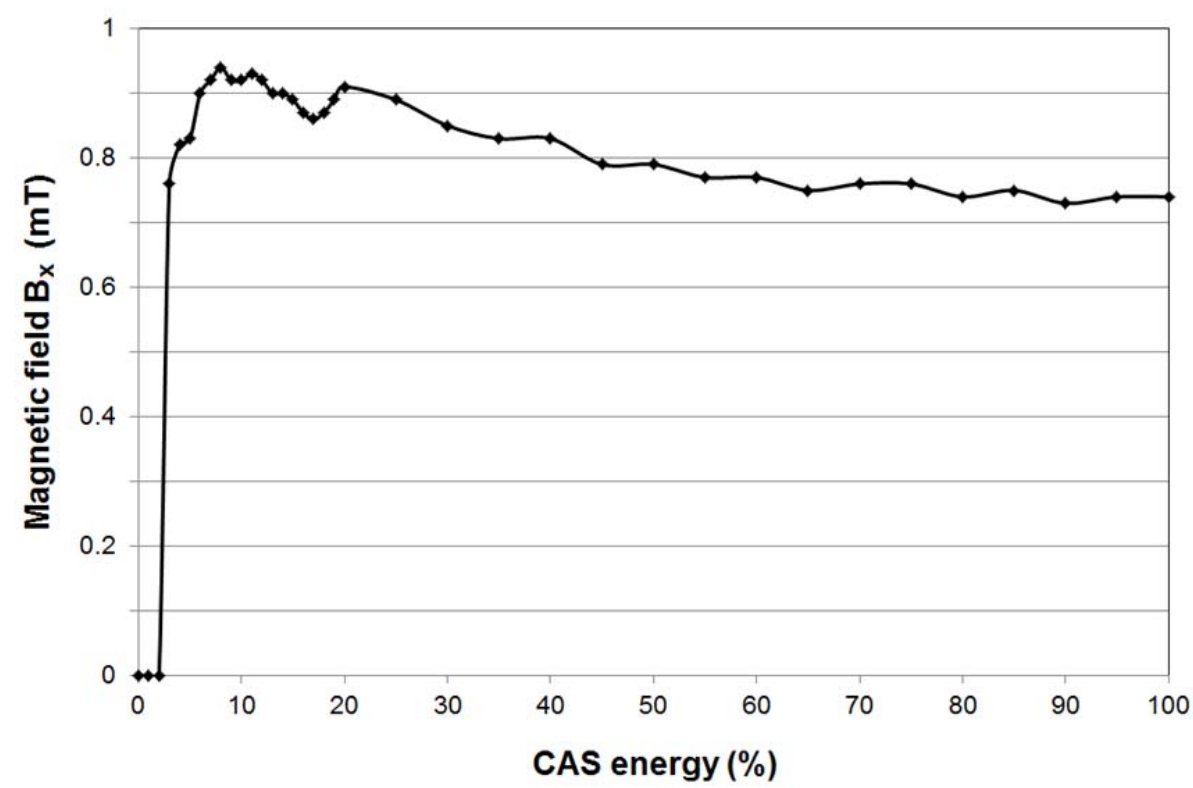

b)

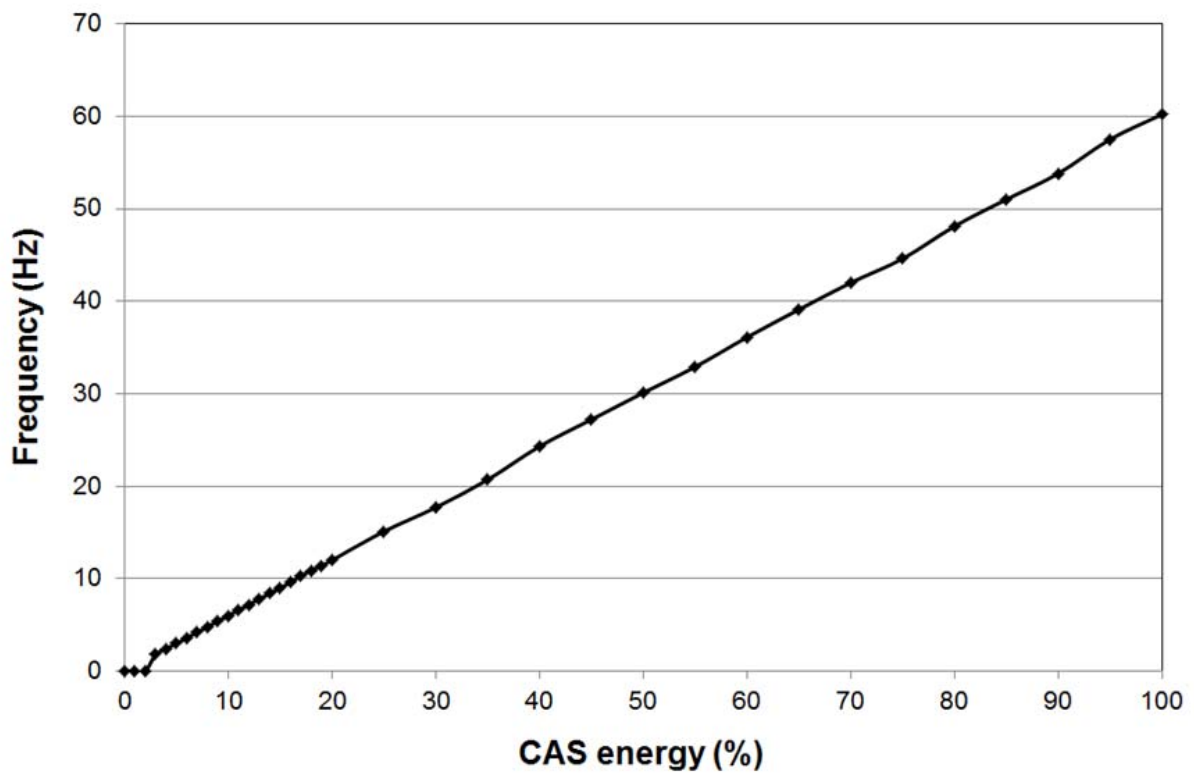


FIGURE 3

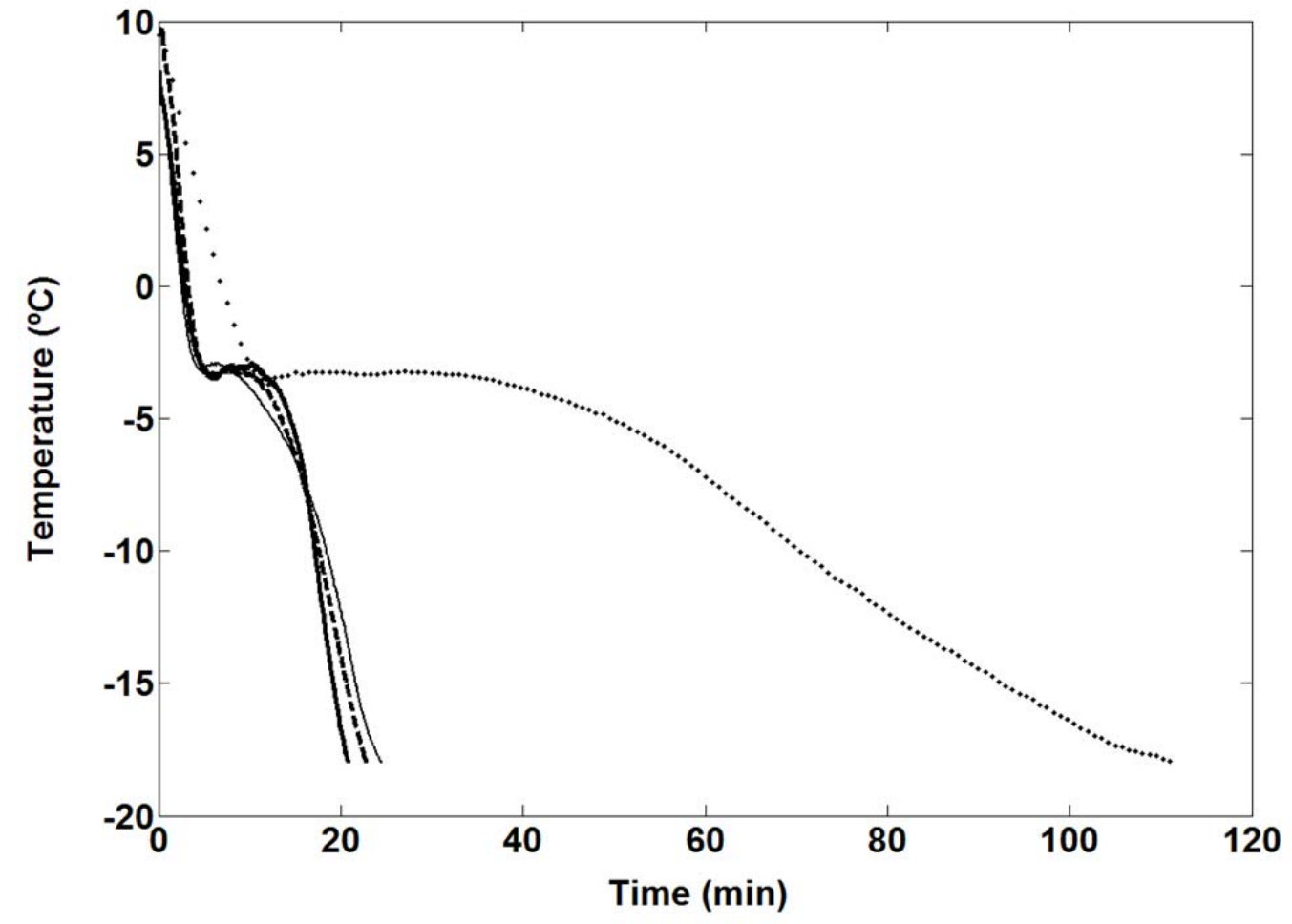




\section{FIGURE 4}

a)

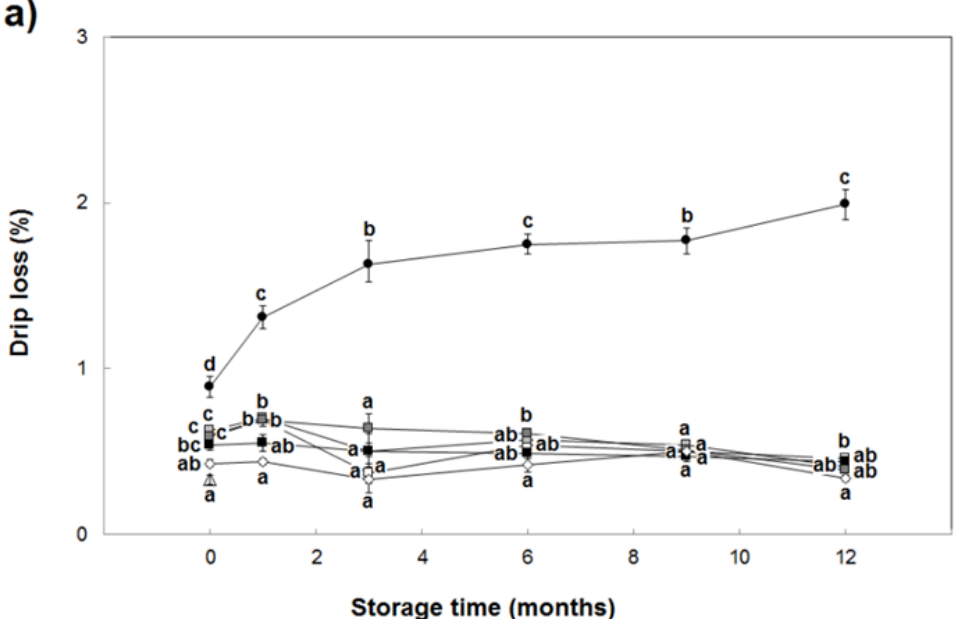

c)

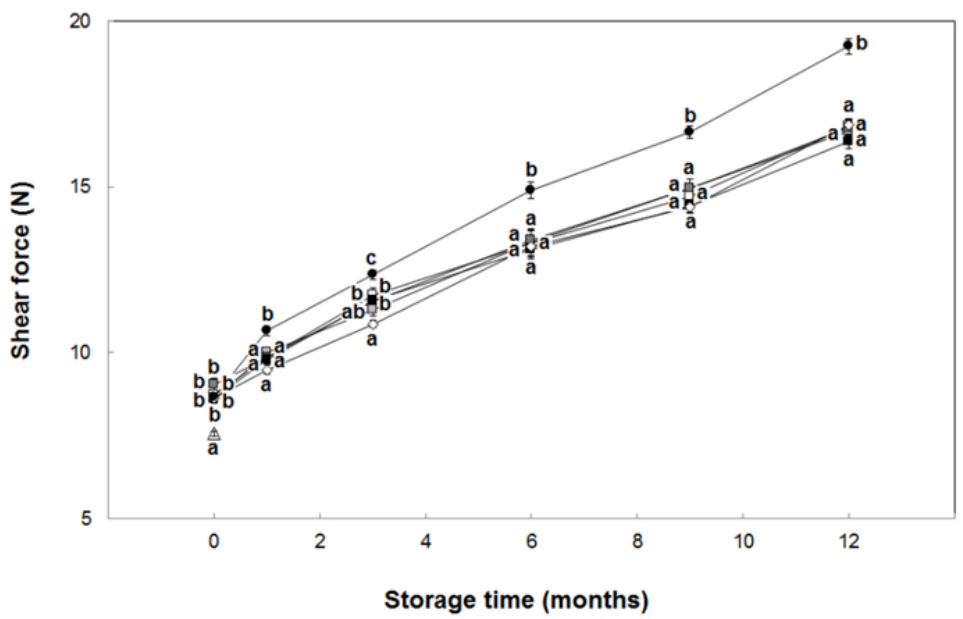

b)

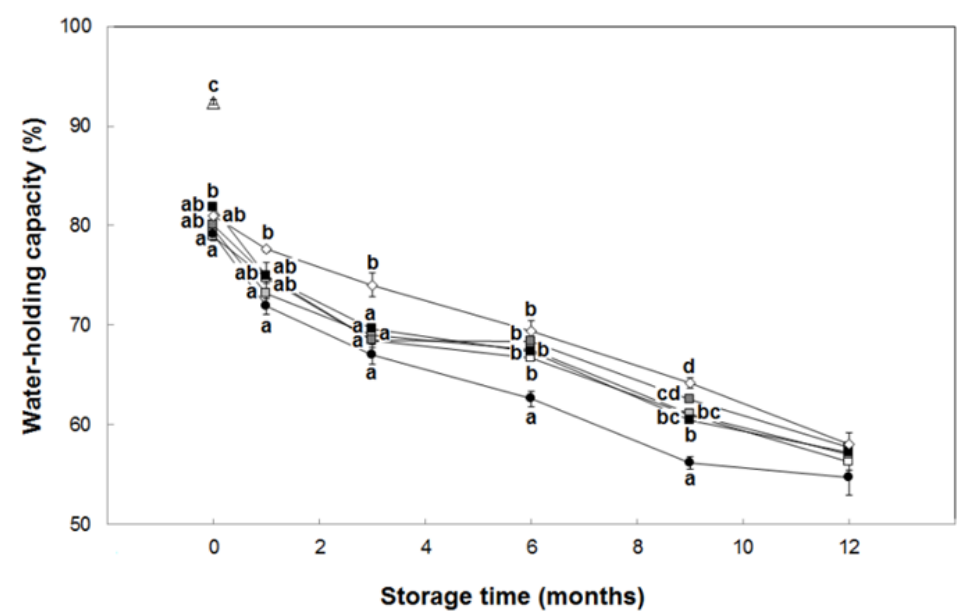

d)

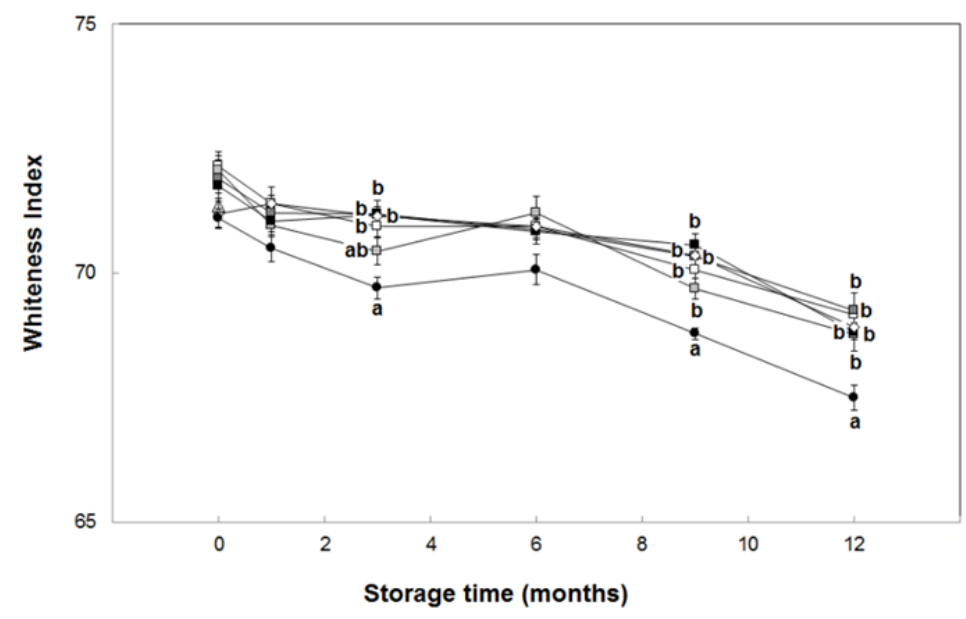


FIGURE 5

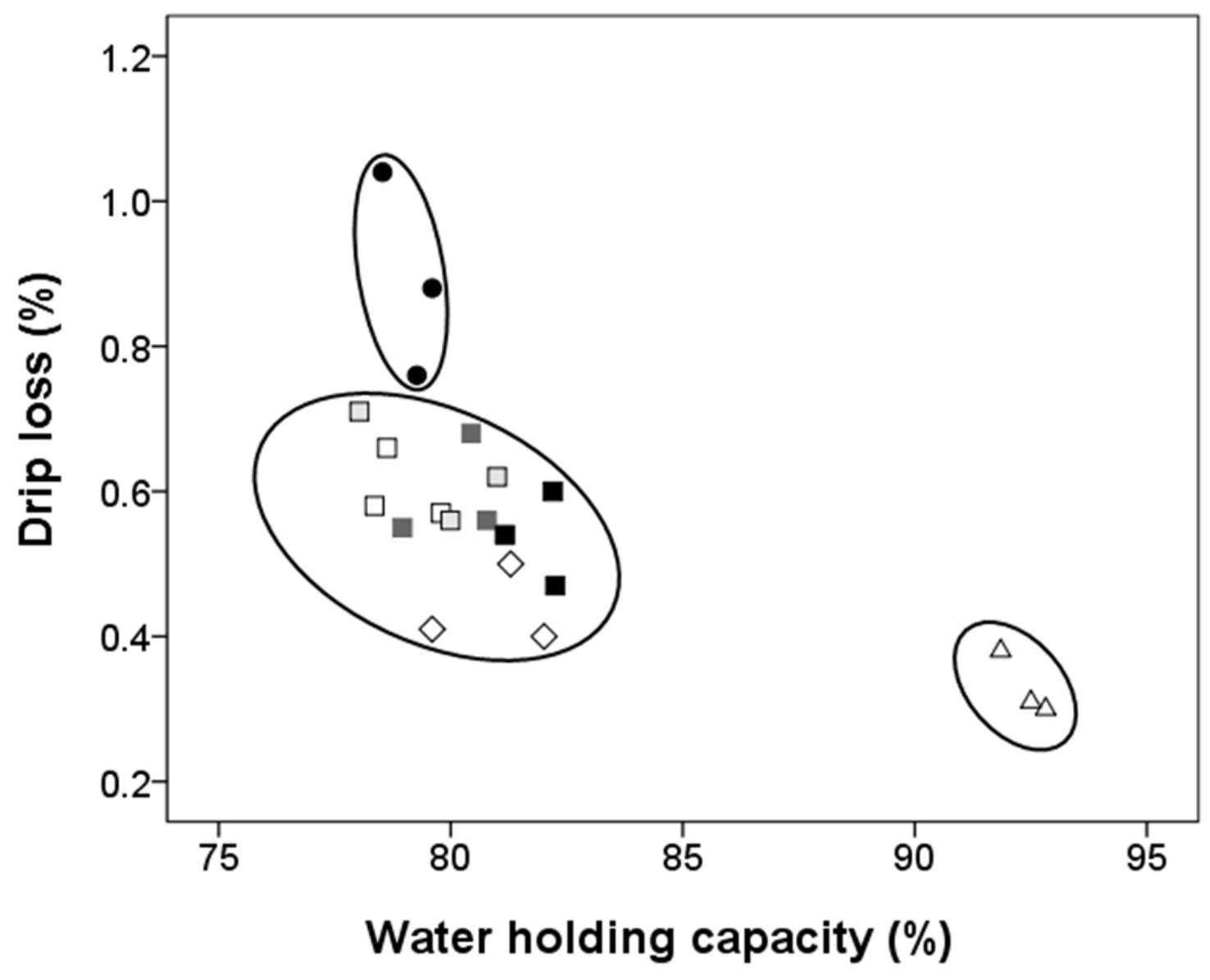

